\title{
Did Dumbo suffer a heart attack? independent association between earlobe crease and cardiovascular disease
}

\author{
Marta Aligisakis ${ }^{1,2^{*}}$, Pedro Marques-Vidal ${ }^{2}$, Idris Guessous ${ }^{3,4}$ and Peter Vollenweider ${ }^{2}$
}

\begin{abstract}
Background: Earlobe crease (ELC) has been associated with cardiovascular diseases (CVD) or risk factors (CVRF) and could be a marker predisposing to CVD. However, most studies studied only a small number of CVRF and no complete assessment of the associations between ELC and CVRF has been performed in a single study.

Methods: Population-based study ( $n=$ 4635, 46.7 \% men) conducted between 2009 and 2012 in Lausanne, Switzerland.

Results: Eight hundred six participants (17.4\%) had an ELC. Presence of ELC was associated with male gender and older age. After adjusting for age and gender (and medication whenever necessary), presence of ELC was significantly $(p<0.05)$ associated with higher levels of body mass index (BMI) [adjusted mean \pm standard error: $27.0 \pm 0.2 \mathrm{vs.} 26.02 \pm 0.07 \mathrm{~kg} / \mathrm{m}^{2}$ ], triglycerides $[1.40 \pm 0.03$ vs. $1.36 \pm 0.01 \mathrm{mmol} / \mathrm{L}]$ and insulin $[8.8 \pm 0.2 \mathrm{vs} .8 .3 \pm 0.1 \mu \mathrm{lU} / \mathrm{mL}]$; lower levels of $\mathrm{HDL}$ cholesterol $[1.61 \pm 0.02$ vs. $1.64 \pm 0.01 \mathrm{mmol} / \mathrm{L}]$; higher frequency of abdominal obesity [odds ratio and (95\% confidence interval) 1.20 (1.02; 1.42)]; hypertension [1.41 (1.18; 1.67)]; diabetes [1.43 (1.15; 1.79)]; high HOMA-IR [1.19 (1.00; 1.42)]; metabolic syndrome [1.28 (1.08; 1.51)] and history of CVD $[1.55(1.21 ; 1.98)]$. No associations were found between ELC and estimated cardiovascular risk, inflammatory or liver markers. After further adjustment on BMI, only the associations between ELC and hypertension [1.30 (1.08; 1.56)] and history of CVD [1.47 (1.14; 1.89)] remained significant. For history of CVD, further adjustment on diabetes, hypertension, total cholesterol and smoking led to similar results [1.36 (1.05; 1.77)].
\end{abstract}

Conclusion: In this community-based sample ELC was significantly and independently associated with hypertension and history of CVD.

Keywords: Earlobe crease, Cardiovascular diseases, Cardiovascular risk factors, Epidemiology, Switzerland

\section{Background}

Cardiovascular disease (CVD) is the most common cause of morbidity and mortality in industrialized countries. Most cardiovascular risk factors (CVRF) involve specific investigations (i.e. total, LDL or HDL cholesterol; glucose) and might not be suitable in low or middle-income countries, where access to health resources is scarce. Thus, the identification of simple clinical signs associated with an increased risk of CVD has drawn some attention. One of these signs is the earlobe crease (ELC), defined as "a deep crease in the lobule portion of the auricle" by S.T. Frank in 1973 [1].

\footnotetext{
* Correspondence: Marta.Aligisakis@unil.ch

${ }^{1}$ Faculty of Medicine, University of Lausanne, Lausanne, Switzerland

${ }^{2}$ Department of Internal Medicine, Lausanne University Hospital (CHUV), Rue du Bugnon 46, 1011 Lausanne, Switzerland

Full list of author information is available at the end of the article
}

Similarly to the heart, earlobes have an end-artery-type of microcirculation without collaterals and become quickly anoxic if end-arteries are occluded [2]. Therefore, CVRF associated with altered microcirculation might impact ELC due to a local micro vascular insufficiency associated with atherosclerosis [2-7].

In cross-sectional studies, ELC was associated with hypertension [8-10], obesity [2, 8], metabolic syndrome (MS) [11], atherosclerosis [8, 12], and coronary artery disease (CAD) $[6,9,10,13-15]$. Various autopsy or biopsy studies also found an association between ELC and systemic atherosclerosis [6, 16-19] or death from CVD [20]. Further, ELC was considered an independent and early sign predisposing to CVD in several prospective studies $[6,10,21-24]$. However, some studies failed 
to find any association between ELC and CAD [25-27] or CVRF $[13,14,21-23,28]$ and two studies suggested that the association of CVD with ELC was entirely accounted for by increasing age [25, 29]. Some of these discrepancies might be related to the fact that most studies focused on a limited number of CVRF and none evaluated in detail the association between ELC and a large panel of established or putative CVRF such as inflammatory markers and adipokines.

Thus, the aim of this study was to assess the associations of ECL with a large panel of CVRF and also with CVD in a population-based sample of adults.

\section{Methods}

\section{Recruitment}

Details of the CoLaus study have been reported previously [30-32]. Briefly, CoLaus is a prospective, population-based study conducted in the city of Lausanne, Switzerland. The main aim is to better understand the prevalence of biological and genetic determinants of CVRF and CVD. The baseline assessment was conducted between 2003 and 2006 and included 6733 participants aged 35 to 75 years. The first follow-up was conducted between April 2009 and September 2012 and included 5064 participants $(75.2 \%$ of the initial sample). Nonparticipation was due to death in 164 (2.4\%) participants, to migration to other countries in 222 (3.3\%), to refusal in $1100(16.3 \%)$ and to non-traceability in $183(2.7 \%)$. The current results are based on the 5064 participants in the first follow-up.

The CoLaus Study was approved by the Institutional Ethics Committee of the University of Lausanne (decision 19 February 2003, protocol number 16/03). All participants provided written informed consent.

\section{Personal history}

Personal history of CAD, angina pectoris, myocardial infarction (MI), stroke and coronary artery bypass graft (CABG) was assessed by questionnaire. Family history included MI and stroke in the mother and/or the father.

Smoking status was self-reported and defined as never, former (irrespective of the time since quitting) and current. Antihypertensive, lipid-lowering and antidiabetes medication was self-reported and defined as present or absent.

Total daily energy expenditure was assessed by a validated questionnaire (PAFQ) where the participant indicated the average daily duration of different types of physical activity (i.e. household, at work, sports) [33]. Energy expenditure was expressed in Kcalories per day and sedentary status was defined as expending less than $10 \%$ of the daily energy in moderate- and high-intensity activities (at least 4 times the basal metabolic rate).

\section{Clinical data}

Earlobe crease, defined as a crease in the lobule portion of the auricle, was systematically searched during the clinical examination by trained field investigators and coded as absent, unilateral or bilateral. A second coding (ELC present/absent) was also used for analysis.

Body weight and height were measured with participants standing without shoes in light indoor clothes. Body weight was measured in kilograms to the nearest $100 \mathrm{~g}$ using a Seca ${ }^{\circ}$ scale (Hamburg, Germany). Height was measured to the nearest $5 \mathrm{~mm}$ using a Seca ${ }^{\circ}$ gauge (Hamburg, Germany). Body mass index (BMI) was defined as weight $(\mathrm{kg}) /$ height $(\mathrm{m})^{2}$.

Waist circumference was measured with a nonstretchable tape over the unclothed abdomen at the narrowest point between the lowest rib and the iliac crest. Two measures were made and the mean, expressed in centimetres, was used for analyses. Abdominal obesity was defined as a waist circumference $>102 \mathrm{~cm}$ (men) and $>88 \mathrm{~cm}$ (women) according to the National Cholesterol Education Program-Adult Treatment Panel III (NCEP-ATP III).

Blood pressure was measured thrice on the left arm, with an appropriately sized cuff, after a rest of at least $10 \mathrm{~min}$ in the seated position using a Omron ${ }^{\circ}$ HEM-907 automated oscillometric sphygmomanometer (OMRON, Japan). The average of the last two measurements was used for analyses. Hypertension was defined as a systolic blood pressure (SBP) $>140 \mathrm{mmHg}$ or a diastolic blood pressure (DBP) $>90 \mathrm{mmHg}$ or presence of antihypertensive medication.

\section{Biological data}

Laboratory markers were measured in venous blood samples drawn after an overnight fast. For cytokine measurements, serum was preferred to plasma because the absolute cytokine level could be influenced by different anticoagulants [34]. Most biological assays were performed by the CHUV Clinical Laboratory on fresh blood samples within $2 \mathrm{~h}$ of blood collection, and additional aliquots were stored at $-80{ }^{\circ} \mathrm{C}$. Most measurements were conducted in a Modular P apparatus (Roche Diagnostics, Switzerland).

Total cholesterol was assessed by CHOD-PAP; highdensity lipoprotein (HDL) cholesterol was assessed by CHOD-PAP + PEG + cyclodextrin; triglycerides were assessed by GPO-PAP; low-density lipoprotein (LDL) cholesterol was calculated using the Friedewald formula if triglycerides were $<4.6 \mathrm{mmol} / \mathrm{L}$.

Glucose was assessed by glucose dehydrogenase; insulin was assessed by a solid-phase, two-site chemiluminescent immunometric assay (Diagnostic Products Corporation, Los Angeles, USA); Homeostatic model assessment insulin resistance (HOMA-IR) was calculated as glucose $(\mathrm{mmol} / \mathrm{L}) \mathrm{x}$ insulin $(\mu \mathrm{IU} / \mathrm{mL}) / 22.5$. High HOMA-IR was defined as $>2.6$. Diabetes was defined as a fasting 
plasma glucose $>7.0 \mathrm{mmol} / \mathrm{L}$ or presence of antidiabetes medication.

Inflammatory markers included high sensitivity-Creactive protein (hs-CRP), Interleukin $1 \beta$ (IL-1 $\beta$ ), interleukin 6 (IL-6) and tumour necrosis factor- $\alpha$ (TNF- $\alpha$ ). Hs-CRP was assessed by immunoassay and latex HS (Immulite 1000-High, Diagnostic Products Corporation, LA, CA, USA) while the other markers were measured using a multiplexed particle-based flow cytometric cytokine assay (Millipore, Zug, Switzerland). Lower detection limits for IL-1 $\beta$, IL- 6 and TNF- $\alpha$ were $0.2 \mathrm{ng} / \mathrm{L}$. As many participants had cytokine levels below detection limits ( $28 \%$ for IL-1 $\beta$ and $4.5 \%$ for IL-6), inflammatory markers were categorized into quartiles and undetectable values were included in the first quartile. Log-transformed values of available levels were also used in the analysis.

Leptin was measured by ELISA (American Laboratory Products Company, Windham, USA). Adiponectin was assessed by ELISA (R\&D Systems, Inc, Minneapolis, USA). Uric acid was assessed by uricase-PAP and creatinine was assessed by the Jaffe kinetic compensated method.

Aspartate aminotransferase (ASAT), alanine aminotransferase (ALAT), $\gamma$-glutamyl transpeptidase $(\gamma$-GT) and alkaline phosphatase were assessed by the optimized standard method according to the International Federation of Clinical Chemistry at $37^{\circ} \mathrm{C}$.

\section{Other variables}

Metabolic syndrome was defined according to the NCEP ATP-III as at least three of the following criteria: 1) waist circumference $>102 \mathrm{~cm}$ (men) and $>88 \mathrm{~cm}$ (women), 2) triglycerides $\geq 1.7 \mathrm{mmol} / \mathrm{L}$ and/or HDL cholesterol $<1.03 \mathrm{mmol} / \mathrm{L}$ (men) or $<1.29 \mathrm{mmol} / \mathrm{L}$ (women) or lipid lowering medication, 3) Blood pressure $\geq 130 / 85 \mathrm{~mm}$ $\mathrm{Hg}$ or antihypertensive medication, 4) Fasting plasma glucose $\geq 5.6 \mathrm{mmol} / \mathrm{L}$ or antidiabetes medication.

Cardiovascular risk was estimated using three different equations: a calibrated SCORE [35], the original Framingham 1998 equation, and a calibrated version of the Framingham 1998 equation for the Swiss population [36].

\section{Statistical analysis}

Statistical analyses were performed using Stata version 13.1 for Windows (Stata Corp, College Station, Texas, USA). Bivariate statistical analyses were performed by Student's t-test for quantitative variables and by chisquare for categorical variables. Results were expressed as mean \pm standard deviation or as number of participants (percentage). Sensitivity, specificity, positive and negative predictive values for presence versus absence of ELC were computed and presented with their $95 \%$ confidence intervals. For quantitative variables, multivariable analyses were performed by ANOVA and the results were expressed as adjusted mean \pm standard error. For categorical variables (excluding smoking), multivariable analyses were performed by logistic regression and results were expressed as odds ratio (OR) and $95 \%$ confidence interval. For smoking, multivariable analyses were performed using multivariate (polytomous) logistic regression and the results were expressed as relative risk ratio (95\% confidence interval). Two models were applied: adjusted for 1) age and gender and 2) age, gender, and BMI. Analyses were also adjusted for medication whenever appropriate. As cardiovascular risk equations already include cardiovascular risk factors, no adjustment was performed for CVRF. For sensitivity analysis, a model similar to 2) but adjusting for waist circumference instead of BMI was applied; similarly, for history of CVD a further adjustment for hypertension, diabetes, total cholesterol and smoking was performed. Statistical significance was assessed for a two-sided test $p$-value $<0.05$.

\section{Results}

\section{Characteristics of participants}

Of the 5064 participants of the first follow-up, 429 (8.5\%) were excluded because of missing data regarding ELC. Excluded participants were older, had a higher SBP and presented more often with hypertension and abdominal obesity than included participants; the other main characteristics and biological markers did not differ between the two groups (Additional file 1: Table S1). The remaining 4635 participants ( $46.7 \%$ men) had a mean age of $57.8 \pm$ 10.5 years and a mean BMI of $26.2 \pm 4.6 \mathrm{~kg} / \mathrm{m}^{2}$. Eight hundred and six (17.4\%) participants had an ELC, of which 373 (46.3\%) unilateral.

\section{Association between ELC and CVRF}

On bivariate analysis, presence of ELC was significantly associated with higher BMI, SBP, DBP, triglyceride, glucose and insulin levels, and with lower HDL cholesterol levels. Presence of ELC was also associated with a higher likelihood of presenting with abdominal obesity, hypertension, diabetes, insulin resistance or metabolic syndrome. Conversely, no association was found between ELC and smoking status, total and LDL cholesterol (Table 1). Sensitivity, specificity, positive and negative predictive values for presence versus absence of ELC regarding cardiovascular risk factors are presented in Additional file 2: Table S2. For all risk factors sensitivity and positive predictive values were low, whereas specificity and negative predictive values were relatively high, namely regarding personal history of CVD (Additional file 2: Table S2).

After multivariable adjustment on age, gender and medication (whenever necessary), the associations of ELC with BMI, abdominal obesity, hypertension, glucose and insulin levels, diabetes, HOMA-IR, and MS remained (Table 2). Similarly, significant associations were found 
Table 1 Bivariate analysis of the association between earlobe crease and selected cardiovascular risk factors (CoLaus study, Lausanne, 2009-2012)

\begin{tabular}{|c|c|c|c|}
\hline Earlobe crease & Absence $(n=3829)$ & Presence $(n=806)$ & $P$-value \\
\hline Men (\%) & $1708(44.6)$ & $455(56.5)$ & $<0.001$ \\
\hline Age (years) & $56.3 \pm 10.3$ & $63.2 \pm 9.7$ & $<0.001$ \\
\hline BMI (kg/m2) & $25.94 \pm 4.53$ & $27.38 \pm 4.85$ & $<0.001$ \\
\hline \multicolumn{4}{|l|}{ BMI categories (\%) } \\
\hline Normal & $1749(46.2)$ & $255(32.2)$ & \\
\hline Overweight & 1448 (38.2) & $349(44.1)$ & $<0.001$ \\
\hline Obese & $592(15.6)$ & $187(23.6)$ & \\
\hline Abdominal obesity (\%) & $1382(36.3)$ & $362(45.2)$ & $<0.001$ \\
\hline \multicolumn{4}{|l|}{ Smoking status (\%) } \\
\hline Never & $1619(42.3)$ & $300(37.2)$ & \\
\hline Former & $1358(35.5)$ & $347(45.1)$ & $<0.001$ \\
\hline Current & $852(22.2)$ & $159(19.7)$ & \\
\hline \multicolumn{4}{|l|}{ Blood pressure status } \\
\hline $\mathrm{SBP}(\mathrm{mm} \mathrm{Hg})$ & $125 \pm 17$ & $131 \pm 19$ & $<0.001$ \\
\hline $\mathrm{DBP}(\mathrm{mm} \mathrm{Hg})$ & $78 \pm 11$ & $79 \pm 11$ & $<0.001$ \\
\hline Hypertension (\%) & 1409 (36.9) & $475(58.9)$ & $<0.001$ \\
\hline \multicolumn{4}{|l|}{ Lipids (mmol/L) } \\
\hline Total cholesterol & $5.70 \pm 1.03$ & $5.68 \pm 1.10$ & 0.66 \\
\hline LDL cholesterol & $3.45 \pm 0.92$ & $3.44 \pm 0.98$ & 0.88 \\
\hline HDL cholesterol & $1.65 \pm 0.47$ & $1.59 \pm 0.47$ & $<0.001$ \\
\hline Triglycerides & $1.34 \pm 0.90$ & $1.47 \pm 0.91$ & $<0.001^{*}$ \\
\hline \multicolumn{4}{|l|}{ Glycaemic status } \\
\hline Glucose (mmol/L) & $5.83 \pm 1.06$ & $6.20 \pm 1.59$ & $<0.001$ \\
\hline Insulin ( $\mu \mid \mathrm{U} / \mathrm{mL})$ & $8.06 \pm 6.82$ & $9.75 \pm 7.74$ & $<0.001^{*}$ \\
\hline Diabetes (\%) & $343(9.0)$ & $147(18.3)$ & $<0.001$ \\
\hline HOMA-IR & $2.21 \pm 2.48$ & $2.86 \pm 2.65$ & $<0.001$ \\
\hline High HOMA-IR (\%) & $925(24.2)$ & $277(34.4)$ & $<0.001$ \\
\hline Metabolic syndrome (\%) & $1070(28.2)$ & $337(42.1)$ & $<0.001$ \\
\hline
\end{tabular}

Results are expressed as number of participants (\%) or as mean \pm standard deviation. Statistical analysis by Student's t-test or by chi-square

$B M I$ body mass index; abdominal obesity and metabolic syndrome are defined by the NCEP ATP-III criteria; hypertension is defined as SBP > 140 or DBP > 90 mm $\mathrm{Hg}$ and/or anti-hypertensive medication; $L D L$ low-density lipoprotein; HDL high-density lipoprotein; HOMA-IR homeostatic model assessment of insulin resistance; high HOMA-IR is defined as $\geq 2.6$; diabetes is defined as fasting plasma glucose $>7.0 \mathrm{mmol} / \mathrm{L}$ and/or anti-diabetes medication

*P-value calculated on log-transformed values

between the number of ELC and BMI, hypertension, HDL cholesterol, insulin levels, HOMA-IR and diabetes (Additional file 3: Table S3 and Additional file 4: Table S4). After further adjustment for BMI, only hypertension remained associated with ELC, while no association was found with the number of ELC. Similar findings were obtained when the analysis was adjusted for waist instead of BMI (Additional file 5: Table S5).

\section{Association between ELC and history or risk of CVD}

The prevalence of ELC was $16 \%$ in participants without any history of CVD and $33.3 \%$ in those with anamnestic CVD $(p$ value $<0.001)$.
On bivariate analysis, the presence of an ELC was associated with personal history of CVD (CAD; MI; angina pectoris; stroke) or CABG, and with a higher CVD risk (Table 3). Most associations with personal history of CVD remained significant after multivariable adjustment, while the associations with CVD risk became non-significant (Tables 4). Similar findings were obtained when the analysis was adjusted for waist instead of BMI (Additional file 6: Table S6) or when the analysis was adjusted for other CVRF (Additional file 7: Table S7). Conversely, no association was found between ELC and family history of CVD (not shown). 
Table 2 Multivariable analysis of the association between earlobe crease and selected cardiovascular risk factors, CoLaus study, Lausanne, 2009-2012

\begin{tabular}{|c|c|c|c|c|c|c|}
\hline \multirow{2}{*}{$\begin{array}{l}\text { Adjusted for } \\
\text { Earlobe crease }\end{array}$} & \multicolumn{3}{|l|}{ Age and gender } & \multicolumn{3}{|c|}{ Age, gender and body mass index } \\
\hline & Absence $(n=3829)$ & Presence $(n=806)$ & $P$-value & Absence $(n=3829)$ & Presence $(n=806)$ & $P$-value \\
\hline $\mathrm{BMI}\left(\mathrm{kg} / \mathrm{m}^{2}\right)$ & $26.0 \pm 0.1$ & $27.0 \pm 0.2$ & $<0.001$ & - & - & - \\
\hline Abdominal obesity & 1 (ref.) & $1.20(1.02 ; 1.42)$ & $<0.05$ & 1 (ref.) & $0.85(0.66 ; 1.09)$ & 0.19 \\
\hline \multicolumn{7}{|l|}{ Smoking status } \\
\hline Never & - & - & - & - & - & - \\
\hline Former & 1 (ref.) & $1.17(0.98 ; 1.40)$ & 0.08 & 1 (ref.) & $1.15(0.96 ; 1.37)$ & 0.14 \\
\hline Current & 1 (ref.) & $1.11(0.89 ; 1.38)$ & 0.34 & 1 (ref.) & $1.16(0.93 ; 1.45)$ & 0.18 \\
\hline \multicolumn{7}{|l|}{ Blood pressure status } \\
\hline $\mathrm{SBP}(\mathrm{mm} \mathrm{Hg})^{\mathrm{a}}$ & $126 \pm 1$ & $126 \pm 1$ & 0.43 & $126 \pm 1$ & $126 \pm 1$ & 0.89 \\
\hline $\mathrm{DBP}(\mathrm{mm} \mathrm{Hg})^{\mathrm{a}}$ & $78 \pm 1$ & $79 \pm 1$ & 0.11 & $78 \pm 1$ & $78 \pm 1$ & 0.53 \\
\hline Hypertension & 1 (ref.) & $1.41(1.18 ; 1.67)$ & $<0.001$ & 1 (ref.) & $1.30(1.08 ; 1.56)$ & $<0.01$ \\
\hline \multicolumn{7}{|l|}{ Lipids $(\mathrm{mmol} / \mathrm{L})^{\mathrm{b}}$} \\
\hline Total cholesterol & $5.70 \pm 0.02$ & $5.68 \pm 0.04$ & 0.69 & $5.70 \pm 0.02$ & $5.68 \pm 0.04$ & 0.53 \\
\hline LDL cholesterol & $3.45 \pm 0.01$ & $3.45 \pm 0.03$ & 0.94 & $3.45 \pm 0.01$ & $3.43 \pm 0.03$ & 0.57 \\
\hline HDL cholesterol & $1.64 \pm 0.01$ & $1.61 \pm 0.02$ & 0.05 & $1.64 \pm 0.01$ & $1.64 \pm 0.01$ & 0.93 \\
\hline Triglycerides & $1.36 \pm 0.01$ & $1.40 \pm 0.03$ & $0.05^{*}$ & $1.36 \pm 0.01$ & $1.36 \pm 0.03$ & $0.65^{*}$ \\
\hline \multicolumn{7}{|l|}{ Glycaemic status } \\
\hline Glucose $(\mathrm{mmol} / \mathrm{L})^{c}$ & $5.88 \pm 0.02$ & $5.96 \pm 0.04$ & 0.06 & $5.88 \pm 0.02$ & $5.92 \pm 0.04$ & 0.36 \\
\hline Insulin $(\mu \mathrm{IU} / \mathrm{mL})^{c}$ & $8.3 \pm 0.1$ & $8.8 \pm 0.2$ & $0.01^{*}$ & $8.3 \pm 0.1$ & $8.4 \pm 0.2$ & $0.78^{*}$ \\
\hline HOMA-IR & $2.29 \pm 0.04$ & $2.47 \pm 0.09$ & 0.07 & $2.30 \pm 0.04$ & $2.33 \pm 0.08$ & 0.72 \\
\hline Diabetes & 1 (ref.) & $1.43(1.15 ; 1.79)$ & $<0.01$ & 1 (ref.) & $1.25(0.99 ; 1.59)$ & 0.07 \\
\hline High HOMA-IR & 1 (ref.) & $1.19(1.00 ; 1.42)$ & $<0.05$ & 1 (ref.) & $0.95(0.78 ; 1.16)$ & 0.62 \\
\hline Metabolic syndrome & 1 (ref.) & $1.28(1.08 ; 1.51)$ & $<0.01$ & 1 (ref.) & $1.03(0.84 ; 1.26)$ & 0.79 \\
\hline
\end{tabular}

For quantitative variables, multivariable analyses were performed by ANOVA and the results are expressed as adjusted mean \pm standard error. For categorical variables (excluding smoking), multivariable analyses were performed by logistic regression and results are expressed as odds ratio ( $95 \%$ confidence interval). For smoking, multivariable analyses were performed using multivariate (polytomous) logistic regression and the results are expressed as relative risk ratio ( $95 \%$ confidence interval) $B M I$ body mass index; SBP systolic blood pressure; $D B P$ diastolic blood pressure; $L D L$ low-density lipoprotein; $H D L$ high-density lipoprotein; $H O M A-I R$ homeostatic model assessment of insulin resistance; high HOMA-IR is defined as a HOMA-IR $\geq 2.6$; abdominal obesity and metabolic syndrome are defined by the NCEP ATP-III criteria; hypertension is defined as SBP $>140$ or DBP $>90 \mathrm{~mm} \mathrm{Hg}$ or anti-hypertensive medication; diabetes is defined as fasting plasma glucose $>7.0 \mathrm{mmol} / \mathrm{L}$ or antidiabetes medication

*P-value calculated on log-transformed values

a ,adjusting for antihypertensive medication

${ }^{b}$,adjusting for hypolipidemic medication

c,adjusting for antidiabetes medication

Similar associations between ELC and personal history of CVD were obtained after splitting the participants with ELC on unilateral and bilateral ELC (Additional file 6: Table S6, Additional file 7: Table S7, Additional file 8: Table S8, Additional file 9: Table S9).

\section{Association between ELC and other markers}

On bivariate analysis, presence of ELC was associated with higher levels of leptin, uric acid, creatinine and liver enzymes, while no association was found with energy expenditure, sedentary status and adiponectin levels (Additional file 10: Table S10). All associations became non-significant after multivariable adjustment (Additional file 11: Table S11) and similar findings were obtained when adjusting for waist instead of BMI (Additional file 12: Table S12).

On bivariate analysis, presence of ELC was positively associated with Hs-CRP and TNF- $\alpha$, but not with IL-1 $\beta$ or IL-6 (Additional file 13: Table S13). After multivariable adjustment, a positive association was found between ELC and IL-1 $\beta$ in quartiles, while no association was found for the other inflammatory markers (Additional file 14: Table S14). Similar results were obtained when the analysis was performed on log-transformed values, except that the association between ELC and Il-1 $\beta$ was no longer significant (Additional file 14: Table S14) or when the analysis was adjusted for waist instead of BMI (Additional file 15: Table S15). 
Table 3 Bivariate analysis of the association between earlobe crease and history or risk of cardiovascular disease, CoLaus study, Lausanne, 2009-2012

\begin{tabular}{llll}
\hline Earlobe crease & Absence $(n=3829)$ & Presence $(n=806)$ & \\
\hline History of & & & \\
Any cardiovascular disease & $244(6.4)$ & $122(15.1)$ & $60(7.5)$ \\
Coronary artery disease & $92(2.4)$ & $30(3.7)$ & $<0.001$ \\
Angina pectoris & $61(1.6)$ & $34(4.2)$ & $<0.001$ \\
Myocardial infarction & $60(1.6)$ & $28(3.5)$ & $<0.001$ \\
Stroke & $61(1.6)$ & $22(2.7)$ & $<0.001$ \\
Coronary artery bypass graft & $33(0.9)$ & $221(27.5)$ & $<0.001$ \\
High cardiovascular disease risk & & $161(20.0)$ & $<0.001$ \\
SCORE recalibrated & $466(12.2)$ & $201(24.9)$ & $<0.001$ \\
Framingham 1998 & $355(9.3)$ & $453(11.8)$ & $<0.001$ \\
Framingham 1998 recalibrated & &
\end{tabular}

\section{Discussion}

To our knowledge, this is the most comprehensive study on the associations between ELC and CVRF. Our results show that ELC is significantly associated with hypertension and history of CVD independently of other risk factors or potential confounders. Our results also suggest that the associations with the other CVRF reported in the literature could be due to confounding by age, gender or BMI.

\section{Association between ELC and CVRF}

Prevalence of ELC was higher in men and increased with age, a finding already reported [10, 11, 13, 26, 37]. A possible explanation is the changes in skin collagen due to aging, which would lead to the development of ELC [2-7]. The higher prevalence of ELC in men could be related to a higher prevalence of smoking, which would accelerate the skin aging process. Still, no associations between ELC and smoking status were found, a finding in agreement with several other studies [2, 7, 8, 21], but not with another [10]. Thus, the higher prevalence of ELC in men remains an open question.

ELC was strongly and linearly associated with higher BMI and abdominal obesity, a finding already reported by two studies [2, 8] but not by another [10]. Interestingly, most associations between ELC and CVRF or CVD were no longer significant after adjusting for BMI, suggesting that ELC might be a stronger marker of obesity than of other CVRF. The fact that the associations between ELC and hypertension, glucose level and history of CAD remained significant after adjusting for BMI also suggests that ELC could be used as a marker of these CVRF.

Table 4 Multivariable analysis of the association between earlobe crease and personal history of cardiovascular disease, CoLaus study, Lausanne, 2009-2012

\begin{tabular}{|c|c|c|c|c|}
\hline \multirow{2}{*}{$\begin{array}{l}\text { Adjusted for } \\
\text { History of }\end{array}$} & \multicolumn{2}{|l|}{ Age and gender } & \multicolumn{2}{|c|}{ Age, gender and BMI } \\
\hline & OR (95\% Cl) & $P$-value & OR $(95 \% \mathrm{Cl})$ & $P$-value \\
\hline Any CVD & $1.55(1.21 ; 1.98)$ & $<0.001$ & $1.47(1.14 ; 1.89)$ & $<0.01$ \\
\hline Coronary artery disease & $1.85(1.30 ; 2.63)$ & $<0.001$ & $1.80(1.26 ; 2.58)$ & $<0.001$ \\
\hline Angina pectoris & $1.28(0.81 ; 2.03)$ & 0.30 & $1.19(0.74 ; 1.90)$ & 0.47 \\
\hline Myocardial infarction & $1.69(1.08 ; 2.64)$ & $<0.05$ & $1.59(1.01 ; 2.51)$ & $<0.05$ \\
\hline Stroke & $1.34(0.84 ; 2.15)$ & 0.23 & $1.30(0.79 ; 2.11)$ & 0.30 \\
\hline CABG & $1.70(0.97 ; 3.00)$ & 0.07 & $1.67(0.93 ; 2.99)$ & 0.09 \\
\hline \multicolumn{5}{|l|}{ High CVD risk } \\
\hline SCORE recalibrated & $1.11(0.88 ; 1.41)$ & 0.37 & $1.10(0.87 ; 1.39)$ & 0.44 \\
\hline Framingham 1998 & $1.09(0.85 ; 1.41)$ & 0.50 & $0.99(0.76 ; 1.28)$ & 0.91 \\
\hline Framingham 1998 r. & $1.15(0.91 ; 1.47)$ & 0.25 & $1.04(0.81 ; 1.34)$ & 0.75 \\
\hline
\end{tabular}

Results are expressed as adjusted odds ratio (95\% confidence interval) for presence relative to the absence of ELC. Statistical analysis by logistic regression $B M I$ body mass index; OR odds ratio; CVD cardiovascular disease; CABG coronary artery bypass graft; high cardiovascular disease risk defined as a 10 -year risk $\geq 5 \%$ for SCORE and as a 10 -year risk $\geq 20 \%$ for Framingham. r. recalibrated 
ELC was positively associated with hypertension, and this association remained significant even after adjusting for age, gender and BMI. Such association had already been pointed out by some reports [8-10] but by another [13]. Contrary to BMI, no linear association between number of ELC and prevalence of hypertension was found.

On bivariate analysis, ELC was associated with diabetes and several markers of insulin resistance such as lower HDL cholesterol and higher glucose, insulin, HOMA-IR and triglycerides levels. Still, all associations became nonsignificant after adjusting for BMI, a finding partly in agreement with other studies [7, 8, 13]. Similarly, the association between ELC and MS was no longer significant after adjusting for BMI, a finding contradicting another study which found a high association between ELC and MS [11]. Overall, our results suggest that the association between ELC and most metabolic markers might be mediated by obesity levels.

\section{Association between ELC and history or risk of CVD}

ELC was significantly associated with personal history of CVD (particularly CAD) independently of age, gender and BMI; adjusting for waist circumference instead of BMI or further adjustment for the main CVRF (hypertension, smoking, total cholesterol and diabetes) led to similar findings. Interestingly, the fact of having bilateral ELC did not lead to a stronger association than having unilateral ELC. Conversely, no association was found between ELC and CVD risk scores after adjusting for age, gender and BMI; the most likely explanation is that age and gender are part of CVD risk equations and that participants with ELC were older, thus leading to an increased CVD risk. Overall, our results suggest that ELC might be related to personal history of CVD but not to absolute CVD risk.

The main hypothesis to explain the association between ELC and CVD is that both organs have an end-artery-type of microcirculation without collaterals [2-7]. Thus, atherosclerotic microvascular changes leading to anoxia in earlobes and to the occurrence of ELC could reflect changes in heart microcirculation. In other words, presence of an ELC would be a marker of defective heart microcirculation. Our findings of an independent association with hypertension which also has a negative impact on the microcirculation are consistent with this hypothesis.

\section{Association between ELC and other markers}

Previous studies have explored the association between ELC and obesity or MS $[2,8,11]$ but data on the associations between ELC and other adiposity markers such as leptin, adiponectin and uric acid are lacking. Our results show that most bivariate associations between ELC and metabolic, adiposity, inflammatory or liver markers were no longer significant in multivariable analysis.
Still, a significant positive association was found between ELC and IL-1 $\beta$, independently of age, gender and BMI or waist. IL-1 $\beta$ is a pro-inflammatory cytokine and has been suggested to be involved in the pathogenesis of atherosclerosis and vascular and myocardial injury [38-40]. However, in the CoLaus study IL- $1 \beta$ levels were not associated with obesity [41].

\section{Study limitations}

This study has several limitations. Due to its crosssectional setting, it was not possible to estimate whether presence of ELC is an independent predictor of CVD. A follow-up study of this cohort is currently under way and will hopefully provide an answer to this issue. Finally, this study is based on a Caucasian population and the results might not be applied to other ethnic groups.

\section{Conclusion}

Earlobe crease is significantly and independently associated with higher prevalence of hypertension and personal history of CVD. The association between ELC and the other metabolic markers appears to be mediated by obesity.

\section{Availability of supporting data}

Researchers using the data are required to follow the terms of a Data Transfer Agreement containing a number of clauses designed to ensure protection of privacy and compliance with relevant laws. For further information go to http://www.colaus.ch/en/cls_home/cls_pro_home/clsresearch-3/cls-agreement.htm. All data requests should be sent to the principal investigator of the CoLaus study.

\section{Additional files}

Additional file 1: Table S1. Comparison between included and excluded participants, CoLaus study, Lausanne, 2009-2012. (PDF 400 kb)

Additional file 2: Table S2. Sensitivity analysis of presence of earlobe crease regarding cardiovascular risk factors and cardiovascular disease, CoLaus study, Lausanne, 2009-2012. (PDF 41 kb)

Additional file 3: Table S3. Bivariate analysis of the association between earlobe crease unilateral or bilateral and selected cardiovascular risk factors, CoLaus study, Lausanne, 2009-2012. (PDF 49 kb)

Additional file 4: Table S4. Multivariable analysis of the association between earlobe crease (unilateral or bilateral) and selected cardiovascular risk factors, CoLaus study, Lausanne, 2009-2012. (PDF 63 kb)

Additional file 5: Table S5. Multivariable analysis of the association between earlobe crease and selected cardiovascular risk factors, CoLaus study, Lausanne, 2009-2012, adjusting for age, gender and waist circumference. (PDF $59 \mathrm{~kb}$ )

Additional file 6: Table S6. Multivariable analysis of the association between earlobe crease and personal history of cardiovascular disease or cardiovascular risk, CoLaus study, Lausanne, 2009-2012, adjusting for age, gender and waist circumference. (PDF $51 \mathrm{~kb}$ )

Additional file 7: Table S7. Multivariable analysis of the association between earlobe crease and personal history of cardiovascular disease, CoLaus study, Lausanne, 2009-2012, adjusted for age, gender, waist 
circumference, total cholesterol, hypertension, diabetes and smoking. (PDF $46 \mathrm{~kb}$ )

Additional file 8: Table S8. Bivariate association between earlobe crease (unilateral or bilateral) and history of cardiovascular disease, CoLaus study, Lausanne, 2009-2012. (PDF 39 kb)

Additional file 9: Table S9. Multivariable association between earlobe crease (unilateral or bilateral) and history of cardiovascular disease, CoLaus study, Lausanne, 2009-2012. (PDF 48 kb)

Additional file 10: Table S10. Bivariate analysis of the association between earlobe crease and physical activity, adipokines or liver markers, CoLaus study, Lausanne, 2009-2012. (PDF 43 kb)

Additional file 11: Table S11. Multivariable analysis of the association between ELC and physical activity, adipokines or liver markers, CoLaus study, Lausanne, 2009-2012. (PDF 54 kb)

Additional file 12: Table S12. Multivariable analysis of the association between earlobe crease and physical activity, adipokines or liver markers, CoLaus study, Lausanne, 2009-2012, adjusted for age, gender and waist circumference. (PDF $55 \mathrm{~kb}$ )

Additional file 13: Table S13. Bivariate association between earlobe crease and quartiles of inflammatory markers, CoLaus study, Lausanne, 2009-2012. (PDF $41 \mathrm{~kb}$ )

Additional file 14: Table S14. Multivariable association between earlobe crease and being in the highest quartile of inflammatory markers, CoLaus study, Lausanne, 2009-2012. (PDF 51 kb)

Additional file 15: Table S15. Multivariable association between earlobe crease and being in the highest quartile of inflammatory markers, CoLaus study, Lausanne, 2009-2012, adjusted for age, gender and waist circumference. (PDF $57 \mathrm{~kb}$ )

\section{Abbreviations}

ALAT: Alanine aminotransferase; ASAT: Aspartate aminotransferase; BMI: Body mass index; CABG: Coronary artery bypass graft; CAD: Coronary artery disease; CVD: Cardiovascular diseases; CVRF: Risk factors; DBP: Diastolic blood pressure; ELC: Earlobe crease; HDL: High-density lipoprotein cholesterol; HOMA-IR: Homeostatic model assessment insulin resistance; hs-CRP: High sensitivity-C-reactive protein; IL-1 $\beta$ : Interleukin 1 $\beta$; IL-6: Interleukin 6; LDL: Low-density lipoprotein cholesterol; MI: Myocardial infarction; MS: Metabolic syndrome; NCEP-ATP III: National Cholesterol Education Program-Adult Treatment Panel III; OR: Odds ratio; SBP: Systolic blood pressure; TNF-a: Tumour necrosis factor-a; $\gamma$-GT: $\gamma$-glutamyl transpeptidase.

\section{Competing interests}

Peter Vollenweider received an unrestricted grant from GlaxoSmithKline to build the CoLaus study. The other authors report no conflict of interest.

\section{Authors' contributions}

MA made part of the statistical analyses and wrote most of the article. PV conceived the study and revised/edited the article. PMV made part of the statistical analyses and revised/edited the article. IG revised the article for important intellectual content. All authors read and approved the final manuscript.

\section{Acknowledgements}

The authors express their gratitude to the participants in the Lausanne CoLaus study and to the investigators who have contributed to the recruitment.

\section{Funding}

The CoLaus study was and is supported by research grants from GlaxoSmithKline, the Faculty of Biology and Medicine of Lausanne, and the Swiss National Science Foundation (grants 33CSCO-122661, 33CS30-139468 and 33CS30-148401).

\section{Author details}

'Faculty of Medicine, University of Lausanne, Lausanne, Switzerland. ${ }^{2}$ Department of Internal Medicine, Lausanne University Hospital (CHUV), Rue du Bugnon 46, 1011 Lausanne, Switzerland. Institute of Social and Preventive Medicine (IUMSP), Lausanne University Hospital, Lausanne, Switzerland. ${ }^{4}$ Department of Community Medicine, Primary Care and Emergency Medicine, Geneva University Hospitals, Geneva, Switzerland.
Received: 15 August 2015 Accepted: 12 January 2016

Published online: 20 January 2016

\section{References}

1. Frank ST. Aural sign of coronary-artery disease. N Engl J Med. 1973;289(6):327-8.

2. Petrakis NL. Earlobe crease in women: evaluation of reproductive factors, alcohol use, and Quetelet index and relation to atherosclerotic disease. Am J Med. 1995;99(4):356-61.

3. Mirić D, Fabijanić D, Giunio L, Eterović D, Culić V, Bozić I, et al. Dermatological indicators of coronary risk: a case-control study. Int J Cardiol. 1998;67(3):251-5.

4. Uliasz A, Lebwohl M. Cutaneous manifestations of cardiovascular diseases. Clin Dermatol. 2008;26(3):243-54.

5. Abdelmalek NF, Gerber TL, Menter A. Cardiocutaneous syndromes and associations. J Am Acad Dermatol. 2002;46(2):161-83. quiz 183-186.

6. Tranchesi Júnior B, Barbosa V, de Albuquerque CP, Caramelli B, Gebara O, Santos Filho RD, et al. Diagonal earlobe crease as a marker of the presence and extent of coronary atherosclerosis. Am J Cardiol. 1992;70(18):1417-20.

7. Shoenfeld Y, Mor R, Weinberger A, Avidor I, Pinkhas J. Diagonal ear lobe crease and coronary risk factors. J Am Geriatr Soc. 1980;28(4):184-7.

8. Celik S, Erdoğan T, Gedikli O, Kiriş A, Erem C. Diagonal ear-lobe crease is associated with carotid intima-media thickness in subjects free of clinical cardiovascular disease. Atherosclerosis. 2007;192(2):428-31.

9. Shmilovich H, Cheng WY, Rajani R, Dey D, Tamarappoo BK, Nakazato R, et al. Relation of diagonal ear lobe crease to the presence, extent, and severity of coronary artery disease determined by coronary computed tomography angiography. Am J Cardiol. 2012;109(9):1283-7.

10. Evrengül $H$, Dursunoğlu D, Kaftan A, Zoghi $M$, Tanriverdi $H$, Zungur $M$, et al. Bilateral diagonal earlobe crease and coronary artery disease: a significant association. Dermatol Basel Switz. 2004;209(4):271-5.

11. Kang EH, Kang HC. Association between earlobe crease and the metabolic syndrome in a cross-sectional study. Epidemiol Health. 2012;34:e2012004.

12. Friedlander AH, Scully C. Diagonal ear lobe crease and atherosclerosis: a review of the medical literature and oral and maxillofacial implications. J Oral Maxillofac Surg Off J Am Assoc Oral Maxillofac Surg. 2010;68(12):3043-50.

13. Wu X-L, Yang D-Y, Zhao Y-S, Chai W-H, Jin M-L. Diagonal earlobe crease and coronary artery disease in a Chinese population. BMC CardiovasC Disord. 2014;14:43

14. Elliott WJ, Karrison T. Increased all-cause and cardiac morbidity and mortality associated with the diagonal earlobe crease: a prospective cohort study. Am J Med. 1991;91(3):247-54.

15. Lucenteforte E, Romoli M, Zagli G, Gensini GF, Mugelli A, Vannacci A. Ear lobe crease as a marker of coronary artery disease: a meta-analysis. Int $J$ Cardiol. 2014;175(1):171-5.

16. Ishii T, Asuwa N, Masuda S, Ishikawa Y, Shimada K, Takemoto S. Earlobe crease and atherosclerosis. An autopsy study. J Am Geriatr Soc. 1990;38(8):871-6.

17. Patel V, Champ C, Andrews PS, Gostelow BE, Gunasekara NP, Davidson AR. Diagonal earlobe creases and atheromatous disease: a postmortem study. J R Coll Physicians Lond. 1992:26(3):274-7.

18. Edston E. The earlobe crease, coronary artery disease, and sudden cardiac death: an autopsy study of 520 individuals. Am J Forensic Med Pathol. 2006; 27(2):129-33.

19. Lichtstein E, Chapman I, Gupta PK, Chadda KD, Smith H, Schwartz I, et al. Letter: Diagonal ear-lobe crease and coronary artery sclerosis. Ann Intern Med. 1976;85(3):337-8.

20. Kirkham N, Murrells T, Melcher DH, Morrison EA. Diagonal earlobe creases and fatal cardiovascular disease: a necropsy study. Br Heart J. 1989;61(4):361-4.

21. Lichstein E, Chadda KD, Naik D, Gupta PK. Diagonal ear-lobe crease: prevalence and implications as a coronary risk factor. N Engl J Med. 1974; 290(11):615-6.

22. Jorde LB, Williams RR, Hunt SC. Lack of association of diagonal earlobe crease with other cardiovascular risk factors. West J Med. 1984;140(2):220-3.

23. Pasternac A, Sami M. Predictive value of the ear-crease sign in coronary artery disease. Can Med Assoc J. 1982;126(6):645-9.

24. Elliott WJ, Powell LH. Diagonal earlobe creases and prognosis in patients with suspected coronary artery disease. Am J Med. 1996;100(2):205-11.

25. Brady PM, Zive MA, Goldberg RJ, Gore JM, Dalen JE. A new wrinkle to the earlobe crease. Arch Intern Med janv. 1987;147(1):65-6.

26. Davis TM, Balme M, Jackson D, Stuccio G, Bruce DG. The diagonal ear lobe crease (Frank's sign) is not associated with coronary artery disease or retinopathy in type 2 diabetes: the Fremantle Diabetes Study. Aust N Z J Med. 2000:30(5):573-7. 
27. Gral T, Thornburg M. Earlobe creases in a cohort of elderly veterans. J Am Geriatr Soc. 1983;31(3):134-6.

28. Kaukola S, Manninen V, Valle M, Halonen PI. Ear-lobe crease and coronary atherosclerosis. Lancet. 1979:2(8156-8157):1377.

29. Mehta J, Hamby RI. Letter: Diagonal ear-lobe crease as a coronary risk factor. N Engl J Med. 1974;291(5):260.

30. Firmann M, Mayor V, Vidal PM, Bochud M, Pécoud A, Hayoz D, et al. The CoLaus study: a population-based study to investigate the epidemiology and genetic determinants of cardiovascular risk factors and metabolic syndrome. BMC Cardiovasc Disord. 2008;8:6.

31. Marques-Vidal P, Bochud M, Bastardot F, von Känel R, Aubry J-M, Gaspoz J-M, et al. Assessing the associations between mental disorders, cardiovascular risk factors, and cardiovascular disease: the Colaus/PsyColaus Study. Raisons Santé IUMSP, 2011; p. 182. http://www.iumsp.ch/Publications/pdf/rds182_fr.pdf.

32. Marques-Vidal P, Paccaud F, Waeber G, Vollenweider P. L'apport de CoLaus en santé publique. Rev Médicale Suisse. 2011;315(39):2118-21.

33. Bernstein M, Sloutskis D, Kumanyika S, Sparti A, Schutz Y, Morabia A. Data-based approach for developing a physical activity frequency questionnaire. Am J Epidemiol. 1998;147(2):147-54.

34. Lee S-A, Kallianpur A, Xiang Y-B, Wen W, Cai Q, Liu D, et al. Intra-individual variation of plasma adipokine levels and utility of single measurement of these biomarkers in population-based studies. Cancer Epidemiol Biomark Prev Publ Am Assoc Cancer Res Cosponsored Am Soc Prev Oncol. 2007; 16(11):2464-70.

35. Marques-Vidal P, Rodondi N, Bochud M, Pécoud A, Hayoz D, Paccaud F, et al. Predictive accuracy and usefulness of calibration of the ESC SCORE in Switzerland. Eur J Cardiovasc Prev Rehabil Off J Eur Soc Cardiol Work Groups Epidemiol Prev Card Rehabil Exerc Physiol. 2008;15(4):402-8.

36. Marques-Vidal P, Rodondi N, Bochud M, Chiolero A, Pécoud A, Hayoz D, et al. Predictive accuracy of original and recalibrated Framingham risk score in the Swiss population. Int J Cardiol. 2009;133(3):346-53.

37. Christoffersen M, Frikke-Schmidt R, Schnohr P, Jensen GB, Nordestgaard BG, Tybjærg-Hansen A. Visible age-related signs and risk of ischemic heart disease in the general population: a prospective cohort study. Circulation. 2014;129(9):990-8

38. Ikonomidis I, Tzortzis S, Lekakis J, Paraskevaidis I, Andreadou I, Nikolaou M, et al. Lowering interleukin-1 activity with anakinra improves myocardial deformation in rheumatoid arthritis. Heart Br Card Soc. 2009;95(18):1502-7.

39. Ikonomidis I, Lekakis JP, Nikolaou M, Paraskevaidis I, Andreadou I, Kaplanoglou T, et al. Inhibition of interleukin-1 by anakinra improves vascular and left ventricular function in patients with rheumatoid arthritis. Circulation. 2008; 117(20):2662-9.

40. Ørn S, Ueland T, Manhenke C, Sandanger $\varnothing$, Godang K, Yndestad A, et al. Increased interleukin-1 $\beta$ levels are associated with left ventricular hypertrophy and remodelling following acute ST segment elevation myocardial infarction treated by primary percutaneous coronary intervention. J Intern Med. 2012; 272(3):267-76.

41. Marques-Vidal P, Bochud M, Bastardot F, Lüscher T, Ferrero F, Gaspoz J-M, et al. Association between inflammatory and obesity markers in a Swiss population-based sample (CoLaus Study). Obes Facts. 2012;5(5):734-44.

\section{Submit your next manuscript to BioMed Central and we will help you at every step:}

- We accept pre-submission inquiries

- Our selector tool helps you to find the most relevant journal

- We provide round the clock customer support

- Convenient online submission

- Thorough peer review

- Inclusion in PubMed and all major indexing services

- Maximum visibility for your research

Submit your manuscript at www.biomedcentral.com/submit

) Biomed Central 